\title{
THE ROLE OF GEOSTATIONARY EARTH ORBIT COMMUNICATION SATELLITES IN CHINESE AREA POSITIONING SYSTEM
}

\author{
Lihua Ma \\ National Astronomical Observatories, Chinese Academy of Sciences, Beijing 100012, China \\ e-mail:mlh@nao.cas.cn
}

\begin{abstract}
The Chinese Area Positioning System (CAPS) is an area positioning system based on Geostationary Earth Orbit (GEO) communication satellites. Transponders on the satellites are used to retransmit navigation message and ranging signals generated from the ground master station, while users receive navigation signals to perform navigation and positioning tasks. Meanwhile, CAPS also develops bidirectional communication receivers using abundant transponders resources on Slightly Inclined Geostationary Orbit (SIGSO) communication satellites, thus realizing integration of navigation and communication in the coverage region.
\end{abstract}

Keywords: Chinese Area Positioning System, Geostationary Earth Orbit, communication satellite, navigation application

\section{INTRODUCTION}

In 1982, Dr. Gerald K. O'Neill had designed the Geostar Satellite System, a commercial network that provided radiodetermination, radiolocation, and radionavigation from the same set of user equipment (O'Neill, 1984). The Geostar Cooperation applied to the Federal Communications Commission (FCC) for a license to broadcast from three Geostationary Earth Orbit (GEO) satellites, which would cover the entire United States in 1983. Mainly due to the rise of the superior Global Positioning System (GPS) which developed fairly, the Geostar Corporation filed for bankruptcy in $1991^{1}$. The China Beidou-1 satellite navigation experimental system, proposed in the 1980s, consists of three GEO satellites and offers limited coverage and applications. The second generation of the system, known as Compass or Beidou-2, which will be a global satellite navigation system consisting of 35 satellites including GEO, Inclined Geosynchronous Orbit (IGSO) and Medium Earth Orbit (MEO) satellites, is still under construction. It is planned to offer services to global users upon its completion in $2020^{2}$. In 2002, a group of Chinese astronomers led by academician Guoxiang Ai presented a new concept, i.e., a navigation system based on GEO communication satellites. The system gave birth to the research and development of the transmitting satellite navigation and positioning system. According to the basic concept of the system, the ground master station measures and computes the signal transmitting time from communication satellites, modulates downlink signals of the satellites to the certain frequency, and furthermore generates and uplinks navigation signals to transponders on the satellites. In comparison to

\footnotetext{
${ }^{1}$ http://en.wikipedia.org/wiki/Gerard_K._O'Neill, 2012-6-19

2 http://en.wikipedia.org/wiki/Beidou_navigation_system, 2012-6-19
} 
GPS, a typical satellite navigation system, there is no need to launch special navigation satellites. Time signals required for navigation are offered by atomic clocks at the ground station, which allows to reduce the developmental and operational period and cost (Ai et al., 2008; Ai et al., 2009b; Li et al., 2010; Ma and Li, 2014).

Under the direction of $\mathrm{Ai}$ et al.'s theoretical frame, research and development for the Chinese Area Positioning System (CAPS) has been carried out since 2003. Extensive positioning experiments within China have been performed since 2005. In the second generation China's satellite navigation system, CAPS is planned to mainly use GEO communication satellites and Slighted Inclined Geostationary Orbit (SIGSO) satellites to compose constellation and offer services to users in the coverage region upon its completion around 2016. (Ma et al, 2011a). The SIGSO satellites are derived from GEO satellites in end-of-life. These satellites can substantially improve the spatial configuration of the constellation and therefore enhance the precision of positioning of this navigation system. Considering that GEO satellites are located near the equatorial plane, they can't be used alone to realize navigation and positioning. An essential improvement is that three-dimension (3D) positioning can be realized with SIGSO satellites (Ai et al., 2008; Ai et al., 2009b; Han et al;, 2009; Shi et al., 2009; Ma, 2011). Besides the irreplaceable role paid by SIGSO satellites in CAPS navigation and positioning applications, the abundant transponders resources on SIGSO satellites can be used to develop communication applications. Therefore, it is feasible and natural to realize the integration of navigation and communication in CAPS, which means the basic services of positioning, velocity and timing (PVT), as well as the communication services, can be provided for users within the region (Shi et al., 2009; Ma, 2011).

In this paper, the role of GEO communication satellites in the CAPS is systematically investigated. Section 2 and section 3 focus on the communication and navigation applications of GEO communication satellites in CAPS respectively. Conclusions are given in the final section of this paper.

\section{NAVIGATION APPLICATIONS}

In the satellite navigation and positioning system, pseudorange measurements from the satellite at epoch $t$ can be modeled as

$$
\rho(\mathrm{t})=\mathrm{r}(\mathrm{t}, \mathrm{t}-\tau)+\mathrm{c}\left[\delta t_{u}(\mathrm{t})-\delta t(\mathrm{t}-\tau)\right]+I(\mathrm{t})+T(\mathrm{t})+\varepsilon_{\rho}(\mathrm{t})
$$

where $r(t, t-\tau)$ is the actual distance between the receiver antenna at signal reception time $t$ and the satellite antenna at signal transmission time $(\mathrm{t}-\tau) ; \delta t_{u}(\mathrm{t})$ and $\delta t(\mathrm{t}-\tau)$ are the receiver and satellite clock offsets, respectively, relative to GPST; the subscript u means the user receiver; $I(\mathrm{t})$ and $T(\mathrm{t})$ are the ionospheric and tropospheric propagation delays, respectively; $\varepsilon_{\rho}(\mathrm{t})$ accounts for modeling errors and unmodeled effects.

The positioning error of the receiver depends upon spatial geometry and pseudorange measurement errors. Giving a simple model of the measurement error in Eq-(2), one can characterize the position accuracy as in Eq-(3) in terms of a Dilution-of-Precision (DOP) parameter and Root-mean-square (RMS) measurement error (Misra and Enge, 2006).

$$
\begin{gathered}
E\left(\varepsilon_{\rho}\right)=0, \quad \operatorname{Cov}\left(\varepsilon_{\rho}\right)=\sigma^{2} I \\
\text { RMS Error }=\sigma \cdot \text { DOP }
\end{gathered}
$$

where $\varepsilon_{\rho}$ denotes the combined effect of the residual measurement errors, $E(\cdot)$ denotes the 
mean value, $\operatorname{Cov}(\cdot)$ denotes covariance, $I$ is identity matrix, and $\sigma$ is the RMS measurement error of a pseudorange measurement. The DOP provides a simple characterization of the quality of the constellation configuration for the satellite navigation system. Obviously, the lower the DOP and $\sigma$, the better the quality of the position estimate, in general. A better positioning system requests DOP values to be small within the effective coverage region at any time. PDOP, HDOP and VDOP are abbreviations for Positional (3D), Horizontal and Vertical DOP. These DOPs can be calculated with the following the formula:

$$
\begin{aligned}
& P D O P=\sqrt{\mathrm{Q}_{11}+\mathrm{Q}_{22}+\mathrm{Q}_{33}} \\
& H D O P=\sqrt{\mathrm{Q}_{11}+\mathrm{Q}_{22}} \\
& V D O P=\sqrt{\mathrm{Q}_{33}}
\end{aligned}
$$

where $\mathrm{Q}_{\mathrm{ii}}(\mathrm{i}=1,2,3,4)$ is the diagonal elements of the precision dilution matrix $\mathrm{Q}=\left(\mathrm{A}^{\mathrm{T}} \mathrm{A}\right)^{-1}, \mathrm{~A}$ is matrix with each row composed of three elements of direction cosine vectors represented in the east-north-up coordinate frame, and an entry of 1 in the last column.

To fully understand the role of GEO satellites in CAPS navigation applications, only two GEO satellites and four SIGSO satellites which are drifted from the GEO satellites by east-west station-keeping (EWSK) maneuvers are employed to constitute the CAPS navigation constellation and achieve the 3D navigation and positioning (Shi et al., 2009; Ma, 2011; Ji et al., 2013). To evaluate the performance of this constellation, time series of the PDOP values covering a full day are calculated for 4 stations, including Beijing, Shanghai, Changchun, and Taibei, based on all visible satellites. In every station, the PDOP value changes with inclination of SIGSO satellites. The PDOP minimum, maximum and average of daily PDOP change are list in Table. 1, Table. 2 and Table. 3, respectively. Here Fig. 1 shows the daily PDOP change under the constellation with $5^{\circ}$ for the SIGSO inclination.

Table. 1. Relationship between minimum of daily PDOP value and the SIGSO inclination

\begin{tabular}{|c|c|c|c|c|}
\hline Station name & $i=3^{\circ}$ & $i=5^{\circ}$ & $i=7^{\circ}$ & $i=9^{\circ}$ \\
\hline Beijing & 24.97 & 15.08 & 10.91 & 8.64 \\
\hline Shanghai & 25.85 & 14.91 & 10.80 & 8.56 \\
\hline Changchun & 26.47 & 16.14 & 11.83 & 9.53 \\
\hline Taibei & 24.47 & 14.80 & 10.73 & 8.51 \\
\hline
\end{tabular}

Table. 2. Relationship between maximum of daily PDOP value and the SIGSO inclination

\begin{tabular}{|c|c|c|c|c|}
\hline Station name & $i=3^{\circ}$ & $i=5^{\circ}$ & $i=7^{\circ}$ & $i=9^{\circ}$ \\
\hline Beijing & 69.84 & 41.96 & 30.05 & 23.46 \\
\hline Shanghai & 69.37 & 41.35 & 29.62 & 23.14 \\
\hline Changchun & 71.53 & 43.14 & 31.03 & 37.16 \\
\hline Taibei & 68.09 & 40.94 & 29.33 & 22.92 \\
\hline
\end{tabular}

Table. 3. Relationship between average of daily PDOP value and the SIGSO inclination

\begin{tabular}{|c|c|c|c|c|}
\hline Station name & $i=3^{\circ}$ & $i=5^{\circ}$ & $i=7^{\circ}$ & $i=9^{\circ}$ \\
\hline Beijing & 39.06 & 23.68 & 17.14 & 13.55 \\
\hline Shanghai & 39.90 & 23.86 & 17.15 & 13.52 \\
\hline Changchun & 41.06 & 24.91 & 18.08 & 15.64 \\
\hline Taibei & 38.73 & 23.32 & 16.84 & 13.29 \\
\hline
\end{tabular}




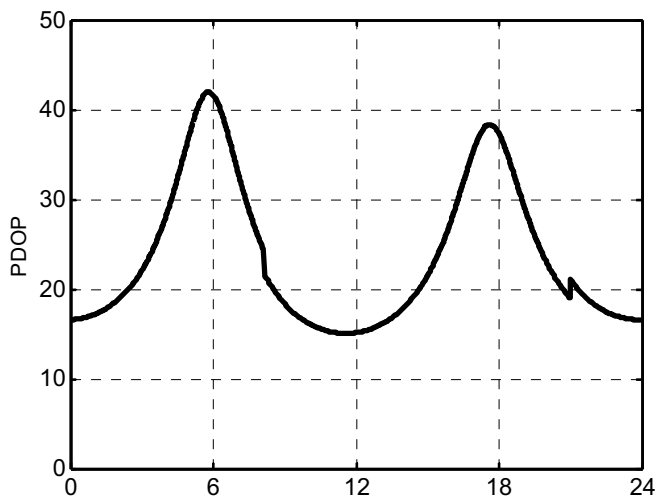

(a)

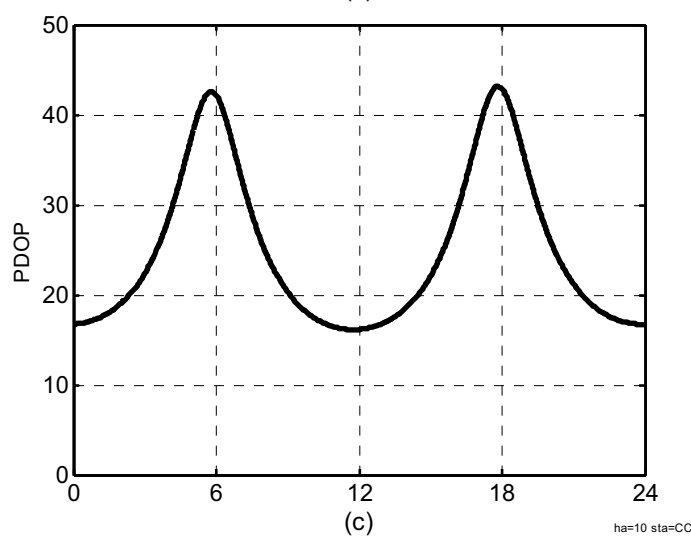

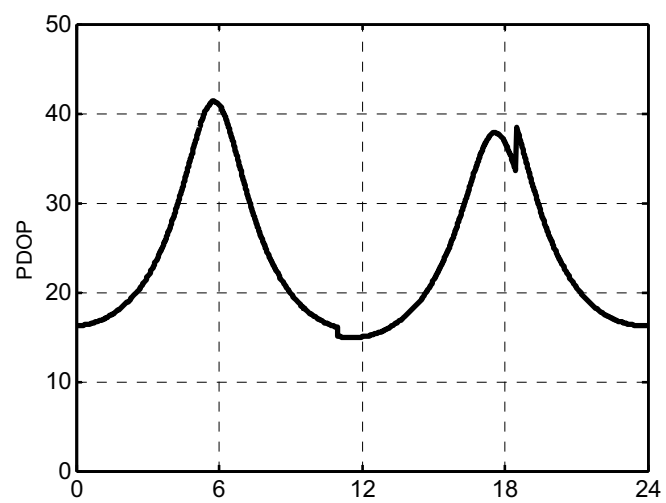

(b)

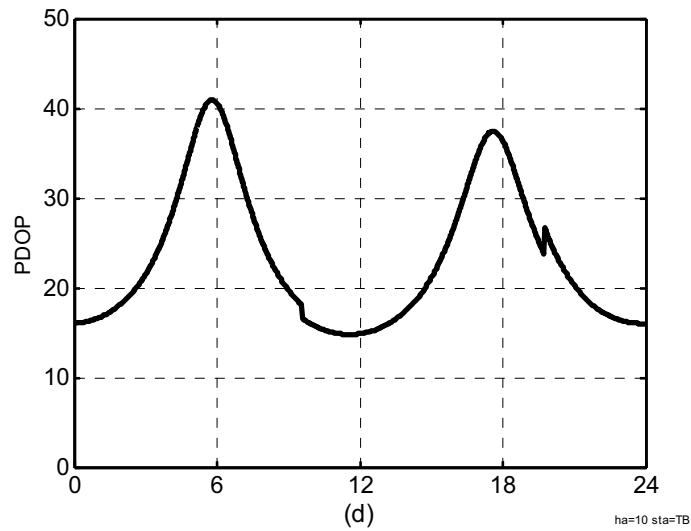

Fig. 1. The daily PDOP change in 4 stations.

The Subfigures (a-d) are corresponding to Beijing, Shanghai, Changchun and Taibei, respectively.

In order to comprehensively analyze the DOP distributions in various areas, the average DOP values over 24 hours are calculated at grid points by latitude and longitude over the coverage area. Fig. 2 and 3 show the regional distribution of daily average HDOP and VDOP numbers, respectively.

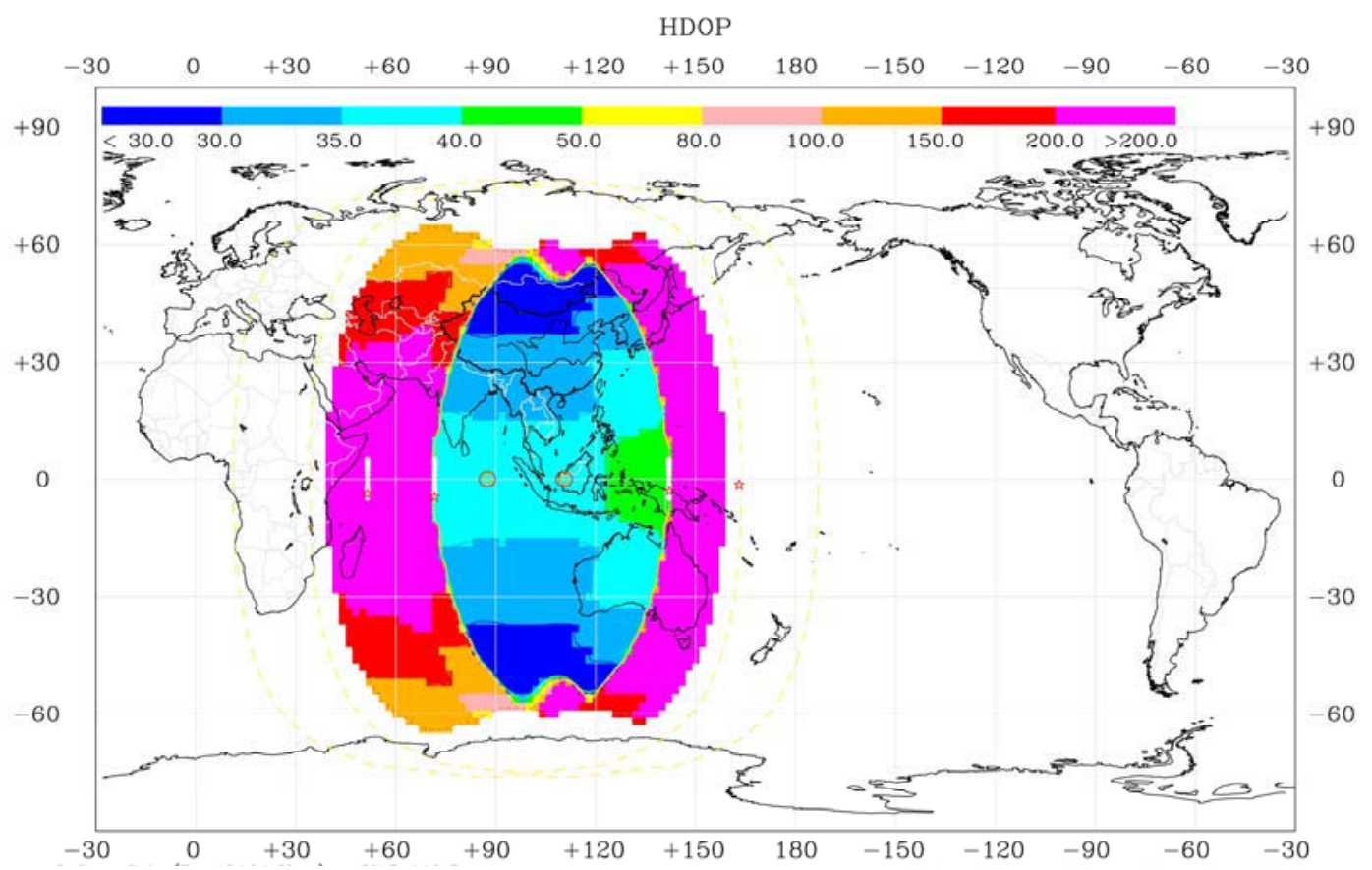

Fig. 2. The average distribution of daily HDOP value 


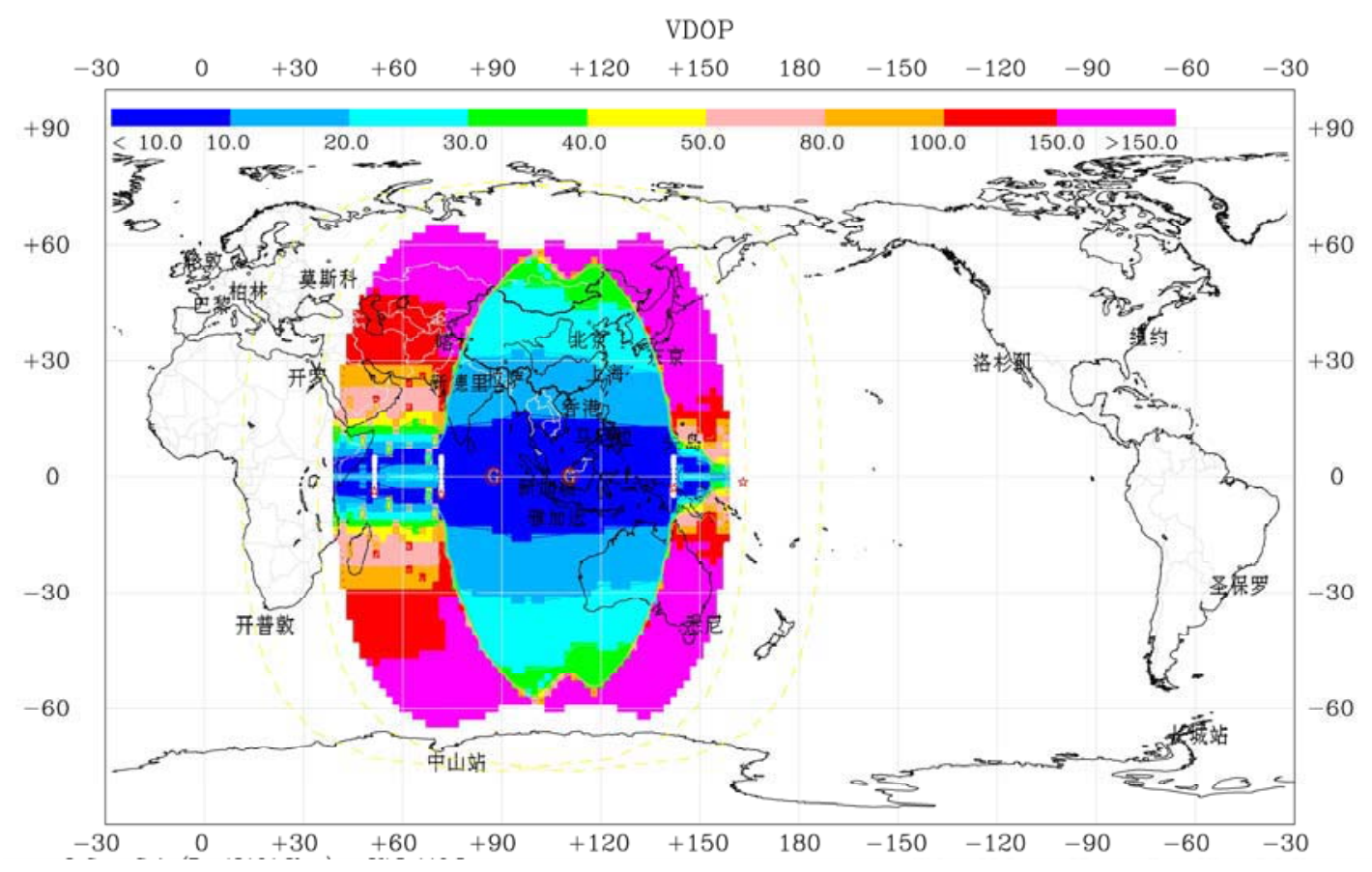

Fig. 3. The average distribution of daily VDOP value

Though the HDOP and VDOP values are not good enough to achieve the navigation and positioning with high accuracy, the concept that a constellation only based on GEO communication satellites can realize the $3 \mathrm{D}$ positioning is brand-new concept and has great importance in the field of research and application. According to the relationship between atmospheric pressure and the altitude, Ai et al. proposed a relative measurement technique in order to obtain local absolute heights and the corresponding principle is given in studies of $\mathrm{Ai}$ et al. (2009a). The user receiver collects local atmospheric pressure and temperature, calculates height relative to the reference station and then constraints the solution in height with altimeter aiding. In the CAPS barometric altimetry system, there are a number of meteorological reference stations all over the country providing local real-time atmosphere parameters. The transponders on communication satellites downlink the meteorological information to the user receiver. The user receiver interpolates the atmosphere parameters and achieves absolute height measurement. In the concept-of-proof phase CAPS uses the barometric altimetry to determine the user's height and further realizes the high accuracy positioning with altitude aiding. The daily average HDOP with barometric aiding is given in Fig. 4. Considering the DOP value is overall smaller, so the scale of HDOP contour figure is reduced. 


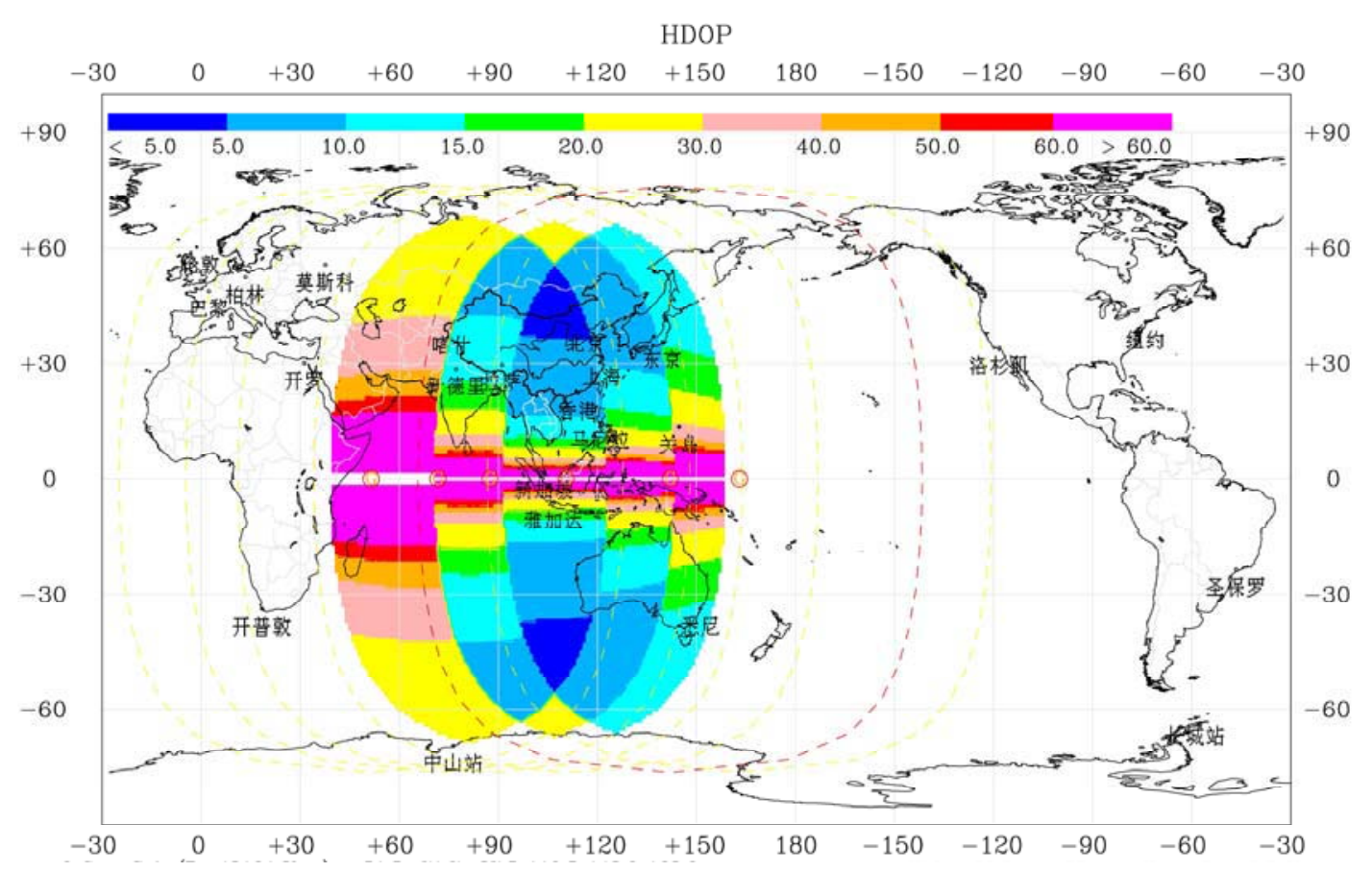

Fig. 4. The average distribution of daily HDOP value with altitude aiding

\section{COMMUNICATION APPLICATIONS}

The GEO communication satellite is maintained in geostationary orbit with up to \pm 0.1 degree precision. However, due to all kinds of perturbations, the GEO satellite is drifting relative to its assumed orbit. In order to control the GEO satellite's drift, one must adjust its orbital position and attitude continually, which will inevitably consume fuel. In order to save fuel, the GEO satellite can be gradually turned into a SIGSO orbit by control of satellite attitude in east-west direction. In these SIGSO satellites three transponders are reserved for navigation purpose, while the remaining transponder resources on these satellites can be used to develop satellite communication techniques and integrate the navigation applications and communication applications (Shi et al., 2009; Ma, 2011; Ma et al., 2011b; Ji et al., 2013).

In an Earth fixed frame the SIGSO orbits constitute a ' 8 ' shape and its drift angle is continually increasing, which poses additional requests to the antenna beam pattern of the user terminal to point any time at the satellite or to cover a very wide range. In such a transmission service, capacities of long-distance transmission in real-time, low amount of information transmission with a strictly low bit error rate and robust anti-interference are required. As there are numerous potential users, the terminals must be cost effective. All those demands constitute the following technical requirements of developing this new kind of satellite communication system.

\section{1) Broadband spread spectrum technique}

As bandwidth resources on SIGSO satellites are abundant, one can trade off frequency bands for power by use of a wideband spread-spectrum communication technique to solve the problem of limited power and to enhance the signal-to-noise ratio. The bandwidths of spread spectrum are optimized in terms of meeting the relevant International Telecommunication Union (ITU) provisions so that we can solve the problem of adjacent satellite interference while using wide beam antenna, reduce the power flux density, and improve the system's capability of anti-jamming. 


\section{2) Large aperture receiving antenna in ground station}

The problem that the small size and wide beam antenna with low gain and power results in limitation of power and Carrier-to-Noise Ratio (CNR) in satellite transmission links. This can be solved by adjusting attenuation in the transponder, or adopting Forward Error Correction (FEC) technique. Besides, a practical solution is to use a large aperture of the receiving antenna at the ground station.

\section{3) Wide shaped-beam antenna}

As mentioned before, SIGSO satellites have daily drifts in ' 8 ' shape. For example, the drift of APSTAR-1A satellite is up to $\pm 7.5^{\circ}$ /day in north-southern direction in March of 2014, and there is a corresponding large change in azimuth and elevation of the satellite observed from the antenna. In the mobile satellite communication systems, the antenna is a crucial component. To some extent, the directing and tracking accuracy of the antenna and its cost restrict the overall performance of the system and thus determine the availability and promotion of the system. To this end, we have developed a monopole shaped-beam antenna. The antenna pattern (shown in Fig. 5) is omnidirectional in azimuth and has wide-beam with about $60^{\circ}$ in elevation. This kind of antenna can well cope with the problems posed by drifts of SIGSO satellites without changing the antenna attitude.

The three techniques mentioned above have special importance for developing this kind of satellite communication system. In addition, some general techniques widely used in satellite communications, such as compression coding, optimized multiple address access, etc., should be optimized and then adopted.

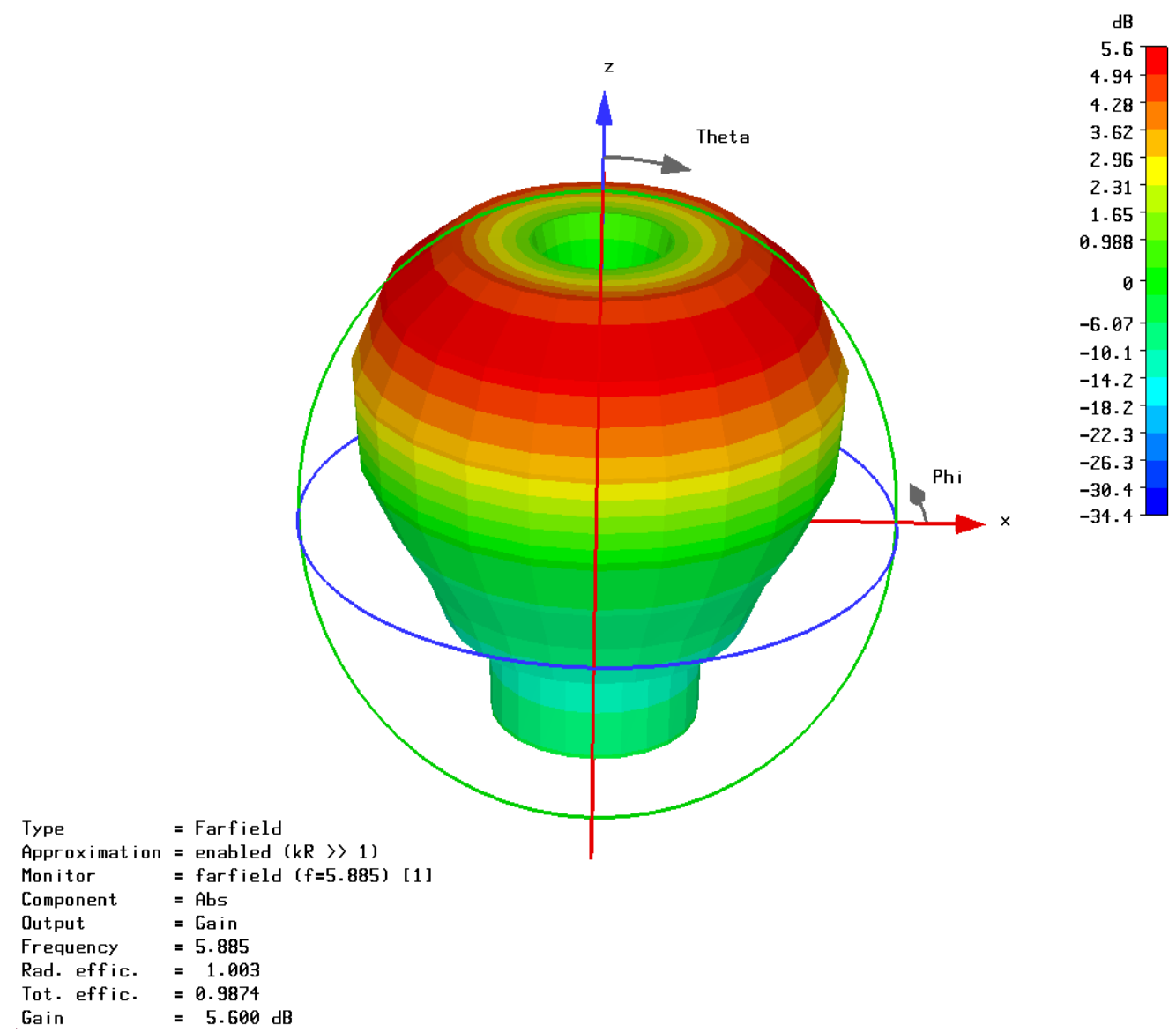

Fig. 5. The 3D pattern of a monopole shaped-beam antenna ${ }^{3}$

\footnotetext{
${ }^{3}$ Courtesy of Senior Engineer Feng Pang, National Astronomical Observatories of China
} 


\section{CONCLUSION AND DISCUSSION}

As CAPS is based on GEO communication satellites, besides providing the basic PVT services, it is also a communication system of large capacity. In the system, navigation and positioning information of individual users can be transmitted timely by transponders on communication satellites to the user center and thus enable the user center to monitor the states of individual users. The system gives impetus to the development of a satellite navigation system from individual navigation to group navigation. By introducing SIGSO satellites, the spatial configuration of the constellation can be fundamentally improved with the essential difference that 3D navigation and positioning can be realized. The RMS error $(\sigma)$ of a pseudorange measurement in CAPS is about 1.0 meter ( $\mathrm{Ji}$ et al., 2013), therefore about 10-20 meters positioning accuracy can be achieved. By aiding with barometric altimetry, the users can reach GPS C/A code positioning accuracy under the constellation of two GEO satellites and four SIGSO satellites in some parts of the coverage area. As plentiful transponder resources on each SIGSO satellites can be employed in communication applications except some of them are reserved for navigation applications. The system has pioneered the study of providing information services based on position, time and state in navigation system, and navigation and communication services. The integration of navigation and communication will certainly enable CAPS with a broad prospect of commercial applications. Therefore, GEO communication satellites, SIGSO satellites specially play a key role in terms of both CAPS navigation and communication applications.

As mentioned above, BeiDou-1 is the Chinese experimental regional navigation system. Its main operation principle includes: (1) the central control station sends inquiry signals to the user via the satellites; (2) when the user terminal received the signal, it sends responding signal back to the satellites; (3) the central station receives the responding signals sent by the user, and calculates the user 2D position; (4) this position is then compared with the digital territorial map stored in the database to obtain the 3D position data; (5) then the position data is sent back to the user via satellites. Different from CAPS, Beidou-1 is not passive positioning system. Its user completes own positioning, while exposing its own position. There are a few users tried to use Beidou-1 to carry out passive position, but ultimately did not reach good results (Liu et al, 2009; Lai, 2009). Therefore the second generation of the system, known as Compass or Beidou-2, has been developing, since 2012 the system has been offering navigation services , mainly for users in China and neighboring regions.

Acknowledgments. The author would like to thank Profs. Guoxiang Ai, Yanben Han, Huli Shi and Xide Cai, who introduced him in satellite navigation study. Dr. Haifu Ji revised the English grammar of the manuscript. The reviewer has given helpful comments, which improved the manuscript greatly. The work was carried out under the support of the National

Basic Research and Development Program of China (Grant No. 2007CB815501), the Key Research Program (Grant No. KJCX2-EW-J01), the Key Research Program of Technology Innovation (KGFZD-125-14-005-2) and the Knowledge Innovation Program (Grant No. KGCX2-EW-407-1) of the Chinese Academy of Sciences.

\section{REFERENCES}

Ai G, Sheng P, Du J, Zheng Y, Cai X, Wu H, Hu Y, Hua Y, Li X. (2009a) Barometric altimetry system as virtual constellation applied in CAPS. Sci China Ser G-Phys Mech Astron, Vol. 52, No. 3, 376-383.

Ai G, Shi H, Wu H, Li Z, Guo J. (2009b) The principle of the positioning system based on communication satellites. Sci China Ser G-Phys Mech Astron, Vol. 52, No. 3, 472-488. 
Ai G, Shi H, Wu H, Yan Y, Bian Y, Hu Y, Li Z, Guo J, Cai X. (2008) Positioning system based satellite communication and Chinese Area Positioning System (CAPS). Chin J Astron Astrophys, Vol. 8, No. 6, 611-630.

Han Y, Ma L, Qiao Q, Yin Z, Shi H, Ai G. (2009) Functions of retired GEO communication satellites in improving the PDOP value of CAPS. Sci China Ser G-Phys Mech Astron, Vol. 52, No. 3, 423-433.

Ji H, Ma L, Ai G, Shi H. (2013) The principle of a navigation constellation composed of SIGSO communication satellites. Research in Astron. Astrophys, Vol. 13, No. 4, 479-489.

Lai D. (2009) Study of Beidou passive positioning. Master's degree thesis, Xidian University

Li, B, Dempster, A. (2010) China's regional navigation satellite system - CAPS. Inside GNSS, Vol. 5, No. 4, 59-63.

Liu J, Lu M, Cui X, Feng Z. (2009) Virtual satellite algorithm for Beidou passive positioning. $J$ Tsinghua Univ (Sci \& Tech), Vol. 49, No. 1, 49-52

Ma L. (2011) The benefits of inclined-orbit operations for geostationary orbit communication satellites. Artificial Satellites, Vol. 46, No. 1, 1-7.

Ma L, Ai G, Ji H. (2011a) A study for IGSO inclination angles in the transmitting satellite navigation constellation. The Journal of Navigation, Vol. 64, No. S1, S73-S82.

Ma L, Li S. (2014) Mathematical aspects for RNSS constellation with IGSO satellites. Earth Science Research, Vol. 3, No. 2, 66-71.

Ma L, Jing Y, Ji H, Zhang L. (2011b) Analysis of evolution of orbits of GEO satellites in end of life based on the STK software. Astronomical Research \& Technology, Vol. 8, No. 4, 347-353.

Misra P., and Enge P. (2006) Global Positioning System: Signals, Measurements, and Performance. Ganga-Jamuna Press.

O'Neill G. (1984) The Geostar System. The Journal of Navigation, Vol. 37, No. 3, 371-373.

Shi H, Ai G, Han Y, Ma L, Chen J, Geng J. (2009) Multi-life cycles utilization of retired satellites. Sci China Ser G-Phys Mech Astron, Vol. 52, No. 3, 323-327.

Received: 2014-03-21,

Reviewed: 2014-07-23, by R. Weber,

Accepted: 2014-08-04. 